\section{Cahiers de Narratologie}

Analyse et théorie narratives

$10.1 \mid 2001$

La voix narrative

\title{
Voix narrative d'une conscience : le cas des écrits spirituels d'Anselme de Cantorbéry
}

Jean-François Cottier

\section{(2) OpenEdition}

1 Journals

\section{Electronic version}

URL: http://journals.openedition.org/narratologie/6957

DOI: 10.4000/narratologie.6957

ISSN: 1765-307X

\section{Publisher}

LIRCES

\section{Printed version}

Date of publication: 1 January 2001

Number of pages: $307-326$

ISBN: 2914561032

ISSN: 0993-8516

\section{Electronic reference}

Jean-François Cottier, "Voix narrative d'une conscience : le cas des écrits spirituels d'Anselme de Cantorbéry", Cahiers de Narratologie [Online], 10.1 | 2001, Online since 28 October 2014, connection on 23 February 2021. URL: http://journals.openedition.org/narratologie/6957 ; DOI: https://doi.org/ 10.4000/narratologie.6957 


\title{
VOIX NARRATIVE D'UNE CONSCIENCE : LE CAS DES ÉCRITS SPIRITUELS D'ANSELME DE CANTORBÉRY
}

\author{
Jean-François COTTIER \\ Université de Nice-Sophia Antipolis
}

Qu'il me soit d'abord permis, en guise d'introduction, d'expliquer en quelques mots le titre et le contenu qu'il recèle. Dans l'expression, « voix narrative d'une conscience ", le mot " voix » est à entendre à la fois à la lettre et au figuré. A la lettre, parce ce que l'on sait que l'expression littéraire médiévale passe par le support nécessaire de la voix ${ }^{1}$, réalité proche de ce que Roland Barthes appellera plus tard l'écriture à haute voix ${ }^{2}$, portée non par des inflexions dramatiques, mais par le grain de la voix, mixte de timbre et de langage. Au figuré également, puisque par métonymie la « voix » pour le moyen âge peut désigner ce que d'autres époques ont appelé plus volontiers «l'écriture »; elle peut aussi, au sens métaphorique de l'expression traditionnelle, désigner les voix intérieures. Quant à la « conscience », si elle peut désigner, comme l'a très bien expliqué M. Zink, le mouvement de réflexivité à l'intérieur de l'activité littéraire sans lequel il peut exister des textes mais non une littérature ${ }^{3}$, elle est à entendre ici davantage comme « conscience religieuse ».

Car c'est bien de textes "religieux", d'œuvres édifiantes et spirituelles, dont il va être question dans cette communication, œuvres que l'on ne saurait enfermer dans une région

1 P. ZUMTHOR, La lettre et la voix. De la "littérature médiévale", Paris, Le Seuil, 1987.

2 R. BARTHES, Le plaisir du texte, Paris, Seuil, 1973 (Points/Essais, $135)$, p. $88-89$.

${ }^{3}$ M. ZINK, La subjectivité littéraire. Autour du siècle de saint Louis, Paris, PUF, 1985, et Les voix de la conscience. Parole du poète et parole de Dieu dans la littérature médiévale, Caen, Paradigmes, p. 7-8. 
secondaire de la littérature médiévale, quand on sait toute l'importance qu'elles avaient pour leur public. La conscience littéraire même et la conscience d'écrire sous le regard de Dieu ne sont en ce temps guère séparables, tant il est vrai qu'alors l'expression personnelle de soi est aussi une défense face à Dieu, comme l'attestent avec force les Confessions d'Augustin, véritable ligne d'horizon de toute la littérature médiévale ${ }^{4}$. Quant aux écrits spirituels d'Anselme de Cantorbéry ${ }^{5}$, textes qui sont au cœur de la présente étude, ils représentent la partie la plus intime de l'œuvre par ailleurs essentiellement philosophique et théologique de celui que les philosophes se plaisent à le considérer comme le «père de la scolastique » et auquel l'Eglise a accordé le titre de Docteur Magnifique, tant sa théologie se déploie dans une langue somptueuse à la logique implacable.

4 On pourrait également citer Guibert de Nogent qui adresse ses Mémoires (Monodia) à Dieu, tout en ouvrant son œuvre par le mot confiteor, allusion directe aux Confessions d'Augustin : GUIBERT DE NOGENT, Autobiographie, éd. et trad. E.-R. LABANDE, Paris, 1981 (CHFMA, 34).

5 Né à Aoste au royaume de Bourgogne en 1033, Anselme se fit moine dans la lointaine Normandie où la réputation de Lanfranc de Pavie, prieur et écolâtre de l'abbaye du Bec, l'avait attiré. Après l'élection de Lanfranc à l'abbatiat, il lui succède comme prieur (1063); quinze ans plus tard il fut lui-même choisi comme abbé (1078), et ce pour quinze autres années. C'est pendant ces trente années de vie monastique que celui que l'Eglise nommera doctor magnificus, consacra son énergie créatrice à la composition d'ouvrages fondamentaux où la méditation théologique et philosophique se fait prière pour dire la foi. Citons les plus célèbres d'entre eux : le Monologion (1076), le Proslogion (1077), et la majorité des prières qui composent le recueil des Prières ou Méditations. L'influence d'Anselme au Bec et le réseau qu'il tissa autour de sa personne fut si considérable qu'on a pu parler à son propos d'un ordo beccensis (R. FOREVILLE). En 1093, à la mort de Lanfranc qui avait accompagné Le Conquérant en Angleterre, Anselme est choisi, malgré ses protestations, pour devenir à son tour archevêque de Cantorbéry. Sa position en tant que primat de l'Eglise d'Angleterre ne fut pas simple, et ses conflits avec les rois Guillaume le Roux et Henri Ier Beauclerc pour défendre la liberté de l'Eglise lui valurent l'exil à deux reprises (10971100 et 1103-1106). Il mourut à Cantorbéry le 21 avril 1109. 
Ce qui caractérise peut-être le plus les prières et méditations anselmiennes, et les différencie de tout ce qui a précédé c'est, outre leur longueur véritablement inhabituelle, leur débordement émotionnel et leur caractère profondément personnel, expression d'une puissante introspection. En termes de signification culturelle, on peut considérer que nous sommes là en présence de l'un des points de départ les plus importants du développement d'une nouvelle spiritualité, attentive à la vie intérieure et qui va développer une dévotion particulière à l'humanité de Christ.

La question du développement de l'expression littéraire du «moi », de " l'ego » a suscité chez les médiévistes de la seconde moitié du $\mathrm{XX}^{\mathrm{e}}$ siècle beaucoup d'intérêt. En particulier, après la publication en 1953 de l'importante étude du professeur anglais R. W. Southern The Making of the Middle Ages dans laquelle il consacre des pages fondamentales au développement de la conscience d'une vie intérieure en Europe entre le $\mathrm{XI}^{\mathrm{e}}$ et le $\mathrm{X} \mathrm{V}^{\mathrm{e}}$ siècle, on a vu paraître de nombreux ouvrages sur la question, dont ceux de Colin Morris $^{6}$, Pierre Courcelle ${ }^{7}$, Walter Ullman ${ }^{8}$, Robert Hanning 9 , et John Benton ${ }^{10}$ pour ne citer que les plus importants d'entre eux. Ces études elles-mêmes, toutes reflet plus au moins conscient de l'idéologie humaniste libérale du temps, ont été remises en cause dans les années 1980 par Caroline Bynum dans son essai intitulé " Did the Twelfth Century discover the Individual ? ", repris et développé dans son célèbre ouvrage Jesus as Mother: Studies in the Spirituality of the High Middle Ages ${ }^{11}$. La savante américaine considère en effet que le

6 The Discovery of the Individual 1050-1200, Londres, SPKC, 1972.

7 Connais-toi toi-même : De Socrate à saint Bernard, Paris, Etudes Augustiniennes, 1975, 3 vol.

8 The Individual and society in the Middle Ages, Baltimore, The Johns Hopkins Press, 1966.

9 The Individual in Twelfth-Century Romance, New Haven, Yale University Press, 1977.

10 Self and Society in Medieval France. The Memoirs of Abbot Guibert of Nogent (1064-1125), edited with an Introduction and Notes, New York-Evanston, Harper and Row, 1970.

11 Berkeley, University of California Press, 1982 : p. 82-109. 
concept même d' « individualité " ou d' " ego " aux XIe-XII siècles n'a rien à voir avec ce que l'on entend par ces mots au $\mathrm{XX}^{\mathrm{e}}$ siècle, "la religion du XII $\mathrm{X}^{\mathrm{e}}$ siècle n'ayant pas exalté la personnalité de l'individu aux dépens de l'appartenance sociale $»^{12}$. Elle réaffirme, ce qui avait déjà été souligné par John Benton, que lorsque l'homme médiéval se tourne vers lui-même il ne s'attend pas à retrouver un " ego » particulier, unique et différent des autres, mais la nature humaine faite à l'image de Dieu, autrement dit le lien qu'il a avec les autres hommes ${ }^{13}$. Dès lors, l'individualisme ne s'oppose pas à l'institution sociale, mais il cherche au contraire à trouver des formes institutionnelles nouvelles et meilleures afin de pouvoir exprimer ses élans religieux intérieurs.

Cela étant donc posé, je voudrais tenter de voir comment les travaux des médiévistes contemporains sur la question, mais aussi les réflexions des philosophes et des linguistes, permettent de relire et de comprendre d'une nouvelle manière les textes spirituels et mystiques d'Anselme, tout à fait novateurs pour leur époque, en nous faisant entendre autrement la voix narrative de la conscience qui s'y exprime.

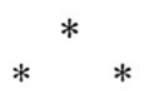

\section{La "révolution" anselmienne}

Dans son premier essai sur saint Anselme, le professeur Southern avait utilisé à propos des écrits spirituels de notre auteur l'expression de "Révolution anselmienne »14; quelque vingt années plus tard il nuança son propos en parlant plutôt

12 Jesus as Mother, p. 85.

13 Jesus as Mother, p. 87.

14 Saint Anselm and his biographer. A study of monastic life and thought 1059-c.1130, Cambridge, 1966, p. 34-47: «Anselmian Revolution ». 
de "Transformation anselmienne $»^{15}$. Pour comprendre la place qu'occupent dans l'évolution de la littérature de dévotion les textes anselmiens, il suffit sans doute d'écouter Anselme lui-même s'exprimant à ce sujet dans une lettre qu'il adresse dès 1072 à la comtesse Adelide, sorte de prologue à son premier recueil de prières et qui contient déjà tout l'esprit de l'entreprise ${ }^{16}:$ " Au sujet des Fleurs des psaumes que votre Grandeur bien-aimée en Dieu a trouvé bon de me commander, notre humilité dans sa fidélité à votre égard n'a pu s'exécuter ni mieux ni plus vite. De fait, notre obéissance a subvenu à votre ordre d'autant plus dévotement que l'ordre lui-même provenait d'une sainte dévotion (...). Le court ouvrage sans valeur que notre pauvre petitesse vous envoie, je demande à votre opulente Excellence de ne pas le dédaigner. Car s'il n'est pas serti d'or et de gemmes, il est toutefois entièrement l'œuvre d'une fidélité affectionnée et le don d'une affection fidèle. A la suite des Fleurs des psaumes, sept Prières ont été ajoutées, dont la première doit moins être appelée Prière que Méditation, car l'âme du pécheur s'y examine rapidement, s'examinant se méprise, se méprisant s'humilie, s'humiliant se trouble à la crainte du jugement dernier, troublée éclate en gémissements et en larmes. En revanche dans les Prières à saint Etienne et à sainte Marie Madeleine, il y a des choses qui, si elles sont dites du fond du cœur lorsqu'on s'y consacre, visent davantage à enflammer l'amour...»17. Ainsi, à la

15 Saint Anselm. A portrait in a landscape, Cambridge, 1991, p. 99106.

16 Voir infra.

17 S. Anselme, Ep. 10, 1. 4-14, éd. F. S. SCHMITT, Sancti Anselmi cantuariensis archiepiscopi opera omnia, Stuttgart-Bad Canstatt, 19842, vol. 3, p. 113 : «De Floribus psalmorum quod dignata est iubere dilecta mihi in Deo uestra Sublimitas, nec citius nec melius potuit exequi fidelis uobis nostra humilitas. Tanto quippe oboedientia nostra imperio uestro deuotius sucessit quanto ipsum imperium a sancta deuotione processit (...). Exiguum et uile munus quod uobis mittit nostra pauper paruitas, rogo ne despiciat uestra diues Nobilitas. Si enim non est auro gemmisque crustatum, est certe totum caritatiua fidelitate factum et fideli caritate donatum. Post Flores psalmorum additce sunt Orationes septem, quarum prima non tantum Oratio quantum Meditatio dicenda est, qua se peccatoris anima breuiter discutiat, discutiendo despiciat, despiciendo humiliet, humiliando terrore ultimi iudicii concutiat, concussa in gemitus 
demande classique de la comtesse d'obtenir de son guide spirituel une sélection d'extraits du psautier, flores psalmorum, Anselme fournit une double réponse. D'une part, en conseiller obéissant, il lui fait parvenir le psautier abrégé demandé - seul ouvrage d'Anselme à n'avoir pas été conservé -, mais d'autre part, il y ajoute, sur une initiative toute personnelle, sept prières de sa composition.

Dans sa lettre dédicatoire, le prieur du Bec ne consacre au psautier que quelques mots ${ }^{18}$ qui font comprendre que le florilège psalmique est avant tout le fruit d'une humble obéissance. En revanche, il analyse en profondeur l'objet de ses Prières, la méthode qu'il convient d'adopter en les lisant, et leur visée. Cette lettre est le premier signe tangible du remplacement progressif des psautiers abrégés, outils privilégiés de la dévotion privée pendant tout le haut moyen âge, par des recueils de prières et de méditations beaucoup plus élaborés et surtout moins impersonnels.

La nouveauté du propos anselmien tient d'une part à la méthode qu'il propose, méthode qui ouvre des chemins nouveaux à la piété personnelle. En effet, bien qu'Anselme n'ait pas véritablement rédigé de traité théorique sur la prière, on peut établir depuis les premiers mots du Proslogion (1077), véritable prière dialectique, jusqu'aux derniers conseils qui servent de Prologue aux Prières ou Méditations, une forme d'ars orandi qui suggère une manière de prier ou de méditer, directement influencée par l'usage des psautiers abrégés : "Les Prières ou Méditations qui suivent, parce qu'elles ont été publiées pour éveiller l'esprit de celui qui lit à l'amour ou à la crainte de Dieu, ou bien à l'examen de soi-même, ne doivent pas être lues dans le tumulte, mais dans le repos, non pas superficiellement et rapidement, mais peu à peu, dans une méditation attentive et qui prend son temps. Le lecteur ne doit pas non plus se proposer de lire jusqu'au bout et entièrement n'importe laquelle des prières, mais seulement autant qu'il sent, avec l'aide de Dieu, que cela lui permet d'aviver le goût

et lacrymas erumpat. In Orationibus uero Stephani et sancta Mariae Magdalena quadam sunt, qua si intimo corde dicantur, cum uacat, plus tendunt ad accendendum amorem ».

${ }^{18}$ Ep. 10, ibidem, 1. 4 et 1. 13, où seul le titre est donné. 
de prier, ou autant qu'il y trouve du plaisir. Il n'est pas nécessaire de les commencer toujours à leur début, mais là où il lui plaira davantage. En effet, des paragraphes les divisent en parties dans l'intention précisément qu'il commence ou qu'il s'arrête à son gré, de peur que la longueur ou la fréquente relecture du même passage n'engendrent l'ennui, et pour qu'au contraire le lecteur y recueille avant tout ce pour quoi elles ont été faites : le goût de la piété ${ }^{19}$.

Dans ce texte inaugural, dont la version finale date des dernières années de la vie d'Anselme, on découvre un enseignement qui désire modifier la vie et l'être de celui qui s'adonne aux exercices spirituels. Mais comme cette transformation intérieure est difficile, elle doit s'opérer peu à peu (paulatim cum intenta et morosa meditatione), et transformer toute la personnalité. On trouve déjà cette volonté affirmée au début du Proslogion : "Et maintenant, pauvre petit homme, dérobe-toi un tout petit peu à tes activités, cache-toi un moment à tes pensées tumultueuses. Rejette maintenant tes

${ }^{19}$ L'CEuvre de S. Anselme de Cantorbéry, Paris, 1988, vol. 5, p. 254 : "Orationes siue Meditationes qua subscripta sunt, quoniam ad excitandam legentis mentem ad Dei amorem uel timorem, seu ad suimet discusionem edita sunt, non sunt legendae in tumultu, sed in quiete, nec cursim et uelociter, sed paulatim cum intenta et morosa meditatione. Nec debet intendere lector ut quamlibet earum totam perlegat, sed quantum sentit sibi, Deo adiuuante, ualere ad accendendum affectum orandi, uel quantum illum delectat. Nec necesse habet aliquam semper a principio incipere, sed ubi magis illi placuerit. Ad hoc enim ipsum paragraphis sunt distinctoe per partes, ut ubi elegerit incipiat aut desinat, ne prolixitas aut frequens eiusdem loci repetitio generet fastidium, sed potius aliquem inde colligat lector propter quod factoe sunt pietatis affectum ". On peut renvoyer également à la lettre 325 (SCHMITT, vol. 5, p. 286) prologue au recueil envoyé à la comtesse Mathilde et qui donne les mêmes conseils de liberté spirituelle, cette lettre pouvant être considérée comme une première rédaction du prologue définitif. De même on peut lire dans la lettre 28 (SCHMITT, vol. 3, p. 136, 1. 15-20) qui accompagne les trois prières à la Vierge qu'Anselme fait parvenir à Gondulfe : «Plaise au Ciel que ces prières soient suffisamment longues pour que, avant qu'on ne parvienne à la fin de chacune d'elles en lisant, ou plutôt en méditant, on y trouve par égard au Ciel, ce pour quoi elles ont été faites : la componction due à la contrition ou à l'amour. Aussi pour cette raison, ai-je voulu les séparer en paragraphes d'après leurs thèmes afin qu'en anticipant l'ennui procuré par leur longueur, on puisse en entamer la lecture où on le souhaite" 
pesants soucis, et remets à plus tard tes pénibles occupations. Vaque un tant soit peu à Dieu, et repose-toi un tant soi peu en Lui. Entre dans la cellule de ton âme, exclus tout hormis Dieu et ce qui t'aide à le chercher, et porte fermée $(M t 6,6)$, cherche-Le. Dis maintenant, tout mon cour, dis maintenant à Dieu : Je cherche ton visage, c'est ton visage, Seigneur, que je recherche $\left(P s\right.$ 26, 8) ${ }^{20}$. Par ailleurs, l'imagination et l'affectivité doivent être elles aussi associées à l'exercice de la pensée - méditation ou prière - et tous les moyens psychagogiques de la rhétorique, toutes les méthodes d'amplification peuvent être ici mobilisées ${ }^{21}$. Car tout exercice spirituel est un combat dont Anselme lui-même connaît la difficulté : "J'ai cherché les biens (Ps 121,9), voici le trouble $(J r 14,19)$ ! Je tendais vers Dieu, je suis tombé sur moi-même. Je cherchais le repos en mon secret, j'ai trouvé tribulation et douleur en mon intime (cf. Ps 114, 3). Je voulais rire de la joie de mon esprit, je suis forcé de rugir du gémissement de mon cœur (cf. Ps 37, 9). L'allégresse était espérée et voici que les soupirs se font denses ! ${ }^{22}$. Rien ne doit donc être négligé pour réussir le chemin d'une conversion devant se réaliser

20 Proslogion, 1, L'Euvre de S. Anselme, vol. 1, p. 97 : «Eia nunc, homuncio, fuge paululum occupationes tuas, absconde te modicum a tumultuosis cogitationibus tuis. Abice nunc onerosas curas, et postpone laboriosas distentiones tuas. Vaca aliquantulum Deo, et requiesce aliquantulum in eo. Intra in cubiculum mentis tua, exclude omnia prater Deum et qua te iunent ad quarendum eum, et clauso ostio quare eum. Dic nunc, totum cor meum, dic nunc Deo : Quaro unltum tuum ; uultum tuum, Domine, requiro ».

21 Voir les pages très éclairantes de P. HADOT à ce sujet, Excercices spirituels et philosophie antique, Paris, 1987, p. 20 sq., et passim; il renvoie lui-même sur ce problème à $\mathrm{P}$. RABBOW, Seelenführung. Methodik der Exerzitien in der Antike, Münich, 1954, p. 55-90; L. HIJMANS, ASKHSIS. Notes on Epictetus educational system, Assen, 1959, p. 89 ; I. HADOT, Seneca und die griechische-römische Tradition der Seelenleitung, Berlin, 1969, p. 17 et 184 et H.-G. INGENKAMP, Plutarchs Schriften über die Heilung der Seele, Göttingen, 1971, p. 99 sq.

22 Proslogion, 1, L'Euvre de S. Anselme, vol. 1, p. 99: «Quasiui bona, et ecce turbatio! Tendebam in Deum, et offendi in me ipsum. Requiem quarebam in secreto meo, et tribulationem et dolorem inueni in intimis meis. Volebam ridere a gaudio mentis mea, et cogor rugire a gemitu cordis mei. Sperabatur latitia, et ecce unde densentur suspiria! ». 
avec la totalité de l'âme ${ }^{23}$. Ce que recherche Anselme c'est la libération du moi de tout ce qui l'enchaîne, afin que par la vraie connaissance de soi il s'ouvre pleinement à la Plénitude elle-même ${ }^{24}$. Et cela il l'opère par le dialogue avec Dieu et avec lui-même, sous la forme de la prière et de la méditation, héritières de la longue tradition des exercices spirituels, philosophiques et religieux, et de la sagesse chrétienne.

Si l'on en vient maintenant au contenu même de ces textes, on peut dire que si l'oratio (la prière) se caractérise surtout par sa dimension dialogique, manifestée par son invocation initiale, la meditatio pour sa part est davantage introspective. C'est ainsi que les trois méditations du recueil (Med 1 "Pour éveiller la crainte »; Med 2 «Déploration sur la malperte de la virginité », Med 3 «Sur la rédemption humaine » $)^{25}$ sont toutes rédigées sous la forme d'un mono-

23 Ce qu'enseignait déjà Platon, République, $518 \mathrm{c}$, édition E. CHAMBRY, Paris, 1961, $(C U F)$, p. 51 : «Comme un œil qu'on ne pourrait tourner de l'obscurité vers la lumière qu'en tournant en même temps tout le corps, la faculté d'apprendre doit être détournée, avec l'âme tout entière, des choses périssables, jusqu'à ce qu'elle devienne capable de supporter la vue de ce qui est... ".

24 C'est l'esprit même de toute la conversion bénédictine, et, plus largement, monastique. C'est ce qui caractérise ce que Grégoire dit de Benoît, Vie et miracles de saint Benoît, 2, 3, 7, in Dialogues, t. 2 : Livres 1-3, édition de A. DE VOGÜE, et traduction de P. ANTIN, Paris, 1979 $(S C, 260)$, p. 144-145: " Je dirai donc que cet homme vénérable habita avec lui-même, parce que toujours en garde et vigilant sur lui-même, se voyant toujours sous l'œil du Créateur, s'examinant toujours, il n'avilit point l'œil de son âme en jetant des œillades à l'extérieur ». C'est à cela que Benoît invite ses fils au début de la Règle, 1, 3: «Ecoute, ô mon fils, l'invitation du maître, et incline l'oreille de ton cœur; recueille avec amour l'avertissement du père qui t'aime, et par tes actes achève-le ; afin de revenir par le labeur de l'obéissance à Celui dont t'avait éloigné la lâcheté de la désobéissance. A toi donc s'adresse maintenant mon discours, qui que tu sois qui renonces à tes propres volontés pour servir sous le vrai roi le Seigneur Jésus-Christ, et prends les armes fortes et glorieuses de l'obéissance... ".

25 Sur le contenu de ces trois textes ainsi que sur celui des prières authentiques, on peut renvoyer aux pages rédigées par dom F.-S. SCHMITT en guise d'introduction à l'édition italienne de G. SANDRI, S. Anselmo d'Aosta. Il Proslogion, le Orazioni e le Meditazioni, Padoue, 
logue se déroulant sous le regard de Dieu : «Âme stérile, que fais-tu ? Pourquoi t'endors-tu, âme pécheresse ?26...Dès à présent c'est lui, dès à présent c'est Jésus. C'est lui le juge entre les mains de qui je tremble...27». Ce mode d'écriture fut certainement influencé par la lecture du De Trinitate d'Augustin, ouvrage qui a profondément marqué Anselme, comme le montre suffisamment l'étude du Monologion, et qu'il connaissait à l'époque où il entreprit la composition de ses premières méditations et prières ${ }^{28}$. C'est précisément l'originalité de la dimension intérieure de la réflexion en présence de Dieu qui a intéressé le Prieur du Bec, et en reprenant le terme de « soliloquia » ${ }^{29}$ qu'Augustin avait forgé pour désigner cette forme nouvelle, Anselme à son tour innove en appelant son premier grand traité Monologion. Lorsqu'il en explique le sens, c'est tout naturellement à l'évêque d'Hippone qu'il renvoie: "J'ai nommé le premier Monologion, c'est-à-dire soliloque... », par opposition au

1959, p. 18-26, ainsi qu'à l'introduction de B. WARD, The Prayers and Meditations of Saint Anselm, with the Proslogion, Londres, 1988, p. 5977.

26 Med 1, 1. 23, L'Ouevre de S. Anselme, vol. 5, p. 77 : «Anima sterilis, quid agis ? Quid torpes, anima peccatrix ?...".

27 Med 1, 1. 80-81, ibidem, p. 79 : «Iam ipse est, iam ipse est lesus. Ipse idem est iudex, inter cuius manus tremo... ».

28 Prologue du Monologion, ibidem, vol. 1, p. 8 : « S'il semble alors à quelqu'un que j'ai avancé, en cet opuscule, quelque point trop nouveau, ou dissonant par rapport à la vérité, qu'il ne crie pas d'emblée, je l'en prie, au novateur présomptueux ou au prôneur de faussetés, mais qu'il scrute d'abord avec soin les livres dudit docteur Augustin sur La Trinité, puis juge, d'après eux, mon opuscule... ".

29 Voir Soliloques 2, 14, texte de l'édition bénédictine, traduction, introduction et notes de P. DE LABRIOLLE, Bruges, 1948 (BA, 5), p. 112-113: (la Raison s'adresse à Augustin) : «Tu as bien tort d'être confus. C'est justement pour t'épargner un pareil sentiment que nous avons choisi ce mode d'entretien. Comme il se déroule entre nous seuls, je voudrais l'appeler et lui donner comme titre "Soliloques" : ce mot est nouveau et peut-être désagréable, mais il dit bien ce qu'il veut dire (nouo quidem et fortasse duro nomine, sed ad rem demonstrandam satis idoneo). Il n'y a pas de meilleur moyen de chercher la vérité que de procéder par demandes et réponses ». 
Proslogion, davantage dialogique, et qui revêt, lui, la forme d'une allocution ${ }^{30}$.

Les thèmes qui reviennent dans les trois méditations sont la crainte de Dieu, l'horreur provoquée par le souvenir du péché, la volonté de guérir ses passions, et surtout le souvenir des bienfaits opérés par le Seigneur : «Homme de rien, laisse donc leur cruauté 31 au jugement de Dieu, et réfléchis à ce que tu dois à ton Sauveur. Considère ce qu'il en était de toi, et ce qui a été fait pour toi. Pense quel est celui qui l'a fait pour toi, de quel amour il est digne. Observe le besoin où tu es, et sa bonté. Vois quelles grâces tu vas rendre, et tout ce que tu dois à son amour. Tu étais dans les ténèbres, tu dévalais la pente surplombant le chaos de l'enfer d'où l'on ne revient pas. Un poids énorme, comme de plomb, pendu à ton cou, t'entraînait. Un fardeau importable t'écrasait, d'invisibles ennemis t'attaquaient de tout leur élan. Ainsi étais-tu sans aucun secours et tu ignorais avoir été conçu et être né en cet état. O qu'en étaitil alors de toi et à qui ces choses te ravissaient-elles ! Crains à t'en souvenir, tremble à y penser... " ${ }^{32}$. Les méditations, par ailleurs, se terminent par une prière qui élève le regard vers Dieu et qui lui adresse les mots ultimes, le monologue s'achevant alors en dialogue ${ }^{33}$.

30 Proslogion, prologue, L'ÉEuvre de S. Anselme, vol. 1, p. 94 : «illud quidem Monologion, id est soliloquium, istud uero Proslogion, id est alloquium nominaui ».

31 Il s'agit de la cruauté des bourreaux du Christ.

32 Med 3, 1. 149-158, ibidem, vol. 5, p. 89 : «Ergo homuncio, illorum crudelitatem dimitte Dei iudicio, et tracta de iis qua debes Saluatori tuo. Considera quid tibi erat et quid tibi factum sit, et pensa qui hoc tibi fecit, quo amore dignus sit. Intuere necessitatem tuam et bonitatem eius, et uide quas gratias reddas et quantum debeas amori eius. In tenebris, in lubrico, indescensu super irremeabile chaos inferni eras. Immensum et quasi plumbeum pondus pendens a collo tuo deorsum te trahebat; onus importabile desuper te premebat; hostes inuisibiles te toto conatu impellebant. Sic eras sine omni auxilio, et nesciebas quia sic conceptus et natus eras. $O$ quid tibi tunc erat, et quo te ista rapiebant! Expauesce memorando, contremisce cogitando...".

33 Voir Med 1, ibidem, vol. 5, p. 79, 1. 86-99: « Jésus, à cause de toimême, sois donc pour moi Jésus "; Med 2, p. 82-83, 1. 86 sq. ; Med 3, p. $91,1.201-211$. 
Enfin, ce qui est nouveau aussi dans le recueil d'Anselme, c'est son esthétique et sa visée. Car si toute théologie chrétienne est touchée au centre par la gloire de la révélation divine, et veut refléter cette impression à partir du centre $^{34}$, celle d'Anselme - qui craint de " traiter d'un si beau sujet dans un style méprisable et sans art $»^{35}$ - est façonnée par une prose rythmée où abondent les antithèses, les parallélismes et les symétries, les jeux de mots et les effets d'accumulation, fort éloignée de l'austère simplicité des textes carolingiens ou anglo-saxons par exemple. Par la beauté dynamique de sa langue, Anselme a ainsi pu donner à l'expression de sa piété une chaleur subtile et généreuse qui frappe l'esprit et le cœur du lecteur et qui l'entraîne irrésistiblement vers la méditation et l'oraison ${ }^{36}$. Son esthétique théologique est donc monastique dans sa forme, "communautaire et dialogale, contemplative, spectatrice, réceptive pour ce qui lui est montré ${ }^{37}$.

Par ailleurs, sa volonté d'indépendance aussi est étonnante puisque, prenant ses distances avec la tradition ancienne, il ne rattache ses prières à aucun ouvrage de dévotion ; son livret existe par lui-même et refuse l'anonymat des recueils antérieurs. On sait en outre, depuis les travaux d'O. Pächt, que c'est Anselme en personne qui a ordonné un programme d'enluminures pour ses prières ${ }^{38}$, fait d'autant plus

34 Voir H. U. Von BALTHASAR, La Gloire et la Croix, Paris, 1968, t. II, p. 11.

$35 \mathrm{CDH}, \mathrm{I}, 3, L^{\prime}$ Eeuvre de S. Anselme, vol. 3, p. 49 : « si tam decoram materiam incompto et contemptibili dictamine exarare prasumo".

36 Idée qu'exprime R. W. SOUTHERN, Anselm, p. 102, lorsqu'il déclare: «We see here (Or 9), therefore, as everywhere in Anselm's writings, a characteristic combination of extreme fervour of expression, systematic completeness, practical restraint. These are the marks of the Anselmian revolution, which we shall observe also in his treatment of friendship : warmth, even violence, of expression is accompanied by great precision of intention and severity of operation ». Voir aussi infra, la comparaison du style d'Anselme et de Rodulfus.

37 H. U. VON BALTHASAR, La Gloire et la Croix, Paris, 1968, t. II, p. 193.

38 O. PÄCHT, «The illustrations of saint Anselm's Prayers and Meditations ", Journal of the Warburg and Courtaud Institutes, 19, 1956, p. 68-83. 
remarquable qu'il s'agirait du premier exemple dans l'histoire de l'enluminure médiévale d'un lien intime entre un auteur et un programme iconographique. Ce que l'on peut distinguer derrière cette double volonté, c'est le désir d'Anselme d'être véritablement un père spirituel guidant ses lecteurs dans leur prière. En outre, si les exercices spirituels étaient jusqu'alors réservés essentiellement au monde monastique, Anselme est de ceux qui en ont ménagé l'accès aux laïcs qui en avaient le désir et la possibilité matérielle. Ainsi de grandes dames de la société de l'époque, comme la comtesse Adélide ou la comtesse Mathilde de Toscane, vont être parmi les premières destinataires de ces textes d'un genre nouveau; elles seront aussi en partie à l'origine de leur diffusion et de leur succès. C'est de cette manière, qu'à l'aube d'un des très grands siècles de la spiritualité occidentale, Anselme accomplit la synthèse du passé tout en ouvrant à l'avenir des chemins nouveaux.

\section{L'expression de soi 39}

Si l'on accepte les Confessions d'Augustin comme horizon à toute expression d'analyse introspective dans la littérature médiolatine, et si l'on se souvient d'autre part que cet ouvrage s'achève sur un commentaire de la Genèse ${ }^{40}$, on comprendra mieux qu'en ce temps l'expérience individuelle se trouve toujours transcendée au niveau même de l'intention profonde du texte. Si par ailleurs, on applique aux textes spirituels d'Anselme les théories contemporaines de philosophes comme Barthes qui a affirmé que l'auteur était mort, de structuralistes, comme Saussure ou Benveniste, pour qui le $J e$ du texte est avant tout une catégorie grammaticale facilement transférable de l'auteur au lecteur, ou de psychanalystes, comme Lacan, qui préfère, plutôt que "d'individu » ou de « personnalité », parler de « sujet » et de « subjectivité »

39 Je suis très redevable pour cette partie aux travaux et aux conseils du professeur américain Thomas Bestul, auteur en particulier d'un communication très suggestive sur cette question : "Self and Subjectivity in the Prayers and Meditations of Anselm of Canterbury ", à paraitre in Actes du Congrès Anselmien de Lublin-Septembre 1996.

40 Aug., Conf., XIII. 
(dans la mesure où il leur semble que la personnalité humaine n'est pas une essence autonome mais un produit dépendant des forces sociales et des circonstances), on ne peut plus penser que les Prières et Méditations peuvent être considérées simplement comme une expression directe de la personnalité d'Anselme, ou d'une vie intérieure parfaitement transmise au lecteur.

Cette perspective nouvelle, et disons-le quelque peu iconoclaste par rapport à la tradition de commentaire de ces textes, a l'intérêt de reconnaître la complexité des relations entre l'auteur, le lecteur et le langage utilisé. L'étude du texte ne part plus de l'auteur ou de ses intentions, mais du langage même : on pourrait dire que ce n'est plus tant Anselme qui crée le langage des prières que le langage des prières qui crée Anselme, c'est-à-dire une voix construite et complexe à laquelle nous nous référons comme étant "Anselme". Une telle approche nous conduit alors à être attentif au statut de la voix que nous entendons dans les prières et à considérer son impact sur le public. On pense en particulier aux complexes dialogues intérieurs qui caractérisent bon nombre de ces textes, en particulier quand le locuteur s'adresse à ses propres péchés, ou au "pauvre homme " (homuncio) comme à un autre personnage, comme dans la Prière à saint Paul: «Malheureux homme de rien, où en est venue ta prière, si se sont évanouies ton espérance et ta confiance ? J'avais commencé à prier avec la confiance de la témérité, je rencontre le désespoir avec l'intelligence de la vérité, et ma prière défaille par désespoir de la moindre bonté envers moi »41. Ou bien quand il utilise la voix des Ecritures pour se faire entendre de Dieu, paroles sacrées elles-mêmes inspirées par Dieu, dans une dynamique réflexive au fondement même de la prière chrétienne ${ }^{42}$ : "Dieu tout-puissant, Père miséricordieux, bon Seigneur, aie pitié de moi pécheur

41 Pr. 10, ibidem, vol. 5, p. 318-319: «Infelix homuncio, ad quid deuenit oratio tua, quo euanuit spes et fiducia tua? Inceperam orare cum fiducia temeritatis, et occurrit desperatio ex intellectu ueritatis, et deficit oratio ex desperatione alicuius erga me pietatis »; cf. aussi le début du Proslogion, ibidem, vol. 1, p. 237.

42 Cf. aussi ibidem, p. $261,265,267$ etc... 
$(L c 18,13) »^{43}$. Par ailleurs, il arrive que la voix de l'orant s'identifie à l'ensemble de la tragédie humaine, l'histoire de la Rédemption n'étant plus le simple rappel d'un récit entendu et connu, mais une expérience vécue personnellement dans la douleur et actualisée sans cesse dans la prière ${ }^{44}$. Enfin, il faut se souvenir des instructions données par Anselme ${ }^{45}$ insistant sur la possibilité d'une lecture fragmentée, accordant leur liberté aux voix individuelles et multipliant les perspectives de ses textes. Ainsi pour reprendre l'extrait de la prière à saint Paul qui vient d'être cité, on peut se demander quelle voix se fait entendre et quelle image d'Anselme est véhiculée, si l'on omet le reste de la composition, et en particulier sa conclusion si consolatrice ? 46

Un autre apport intéressant du post-structuralisme pour la compréhension des Prières d'Anselme est la notion d'exemplarité : un texte, même une prière, exprime moins les pensées intimes d'un auteur qu'il ne construit par sa fonction rhétorique un exemplum parlant auquel un public peut répondre ou sur lequel il peut calquer sa conduite. Cet exemplum ne doit pas être considéré par rapport à sa conformité vis-à-vis de son auteur, mais par rapport à sa réalisation. Il doit donc avant tout être considéré comme une construction linguistique. Il est clair que l'Anselme qui énonce ses prières a la puissance d'un exemple moral et qu'il modèle ceux qui le lisent. Lorsque l'Anselme des Prières pleure sur ses péchés,

43 Pr. 1, ibidem, vol. 5, p. 259 : «Omnipotens Deus et misericors Pater et bone Domine, miserere mihi peccatori ».

44 Cf. Pr. 8, ibidem, vol. 5, p. 301, 1. 23 sq.

45 Cf. supra.

46 Pr. 10, ibid., vol. 5, p. 331 : «Christ, Toi qui es mère et, sous tes ailes, rassemble tes petits, ce mort, ton petit, se glisse sous tes ailes. Car ta douceur conforte les effrayés, ton parfum restaure les désespérés. Que ta chaleur vivifie les morts, que ton toucher justifie les pécheurs? Reconnais, mère, ton fils mort soit au signe de la croix soit à la voix de ta confession. Ranime ton petit, ressuscite ton mort, justifie ton pécheur. Effrayé par Toi, qu'il soit consolé par Toi : désespérant de soi, qu'il soit conforté par Toi et par Toi, reformé dans ta grâce intègre et inaliénable. De toi s'écoule la consolation des malheureux ; béni sois-Tu dans les siècles des siècles. Amen " 
prie pour son pardon et accepte la grâce de Dieu, il entraîne le lecteur dans une démarche similaire. On possède d'ailleurs un témoignage contemporain de ce phénomène, dans une lettre envoyée vers 1076, au début donc de l'élaboration du recueil, à Anselme par Durand, l'abbé de La Chaise-Dieu, qui décrit sa propre réaction à la lecture de la première Méditation d'Anselme : «La piété de la prière que tu as écrite éveille en nous la piété d'une componction endormie, si bien que nous nous réjouissons ensemble en nous élevant par l'esprit, en aimant ces choses en toi, ou plutôt toi en elles, et par dessus elles et avec elles en aimant Dieu et toi ! ${ }^{47}$. Si l'on ajoute à cela que cette lettre de Durand nous est parvenue par les bons soins d'Anselme qui a composé en personne ses recueils de correspondance, on peut remarquer d'une part qu'il était luimême conscient de la valeur d'exemplarité de ses textes, et d'autre part, que la lettre devient elle-même, au second degré, un nouvel exemplum moral pour son lecteur!

Enfin, le post-structuralisme en attaquant la position centrale de l'auteur, que la critique classique a toujours privilégiée pour comprendre ce qu'il est convenu d'appeler le "sens du texte", nous a fourni de nouvelles clés pour entendre les textes. Hans Robert Jauss ${ }^{48}$ a particulièrement insisté sur l'importance du lecteur dans la réception d'un texte, en montrant que ce dernier n'est pas le simple réceptacle passif d'un sens constitué et contrôlé par l'auteur, mais qu'il représente une entité historiquement conditionnée, participant activement à la formation d'une compréhension qui peut donc évoluer au cours du temps. Ce point de vue s'applique particulièrement bien aux textes spirituels d'Anselme, pour lesquelles les lettres et le prologue qui les encadrent encouragent justement le lecteur à une autonomie véritable en la matière en reconnaissant son importance dans la réception et la compréhension de ces écrits. On peut même

47 Ep. 70, éd. SCHMITT, vol. 3, p. 190-191 : «Pietas scriptee tuce orationis excitat in nobis pietatem sopita compunctionis, adeo ut quasi mente prosiliendo congaudeamus, ea diligendo in te, uel potius in eis te, super ea et per ea Deum et te ».

48 Pour une esthétique de la réception, préface de J. STAROBINSKI, Paris, 1978, p. 46-63. 
aller jusqu'à considérer que l'auteur se détache de ses textes en autorisant, contrairement à son usage pour ses autres traités ${ }^{49}$, une lecture aléatoire et libre. Le lecteur en choisissant le passage qui lui convient et le temps qu'il veut lui consacrer recrée d'une certaine façon son propre texte en s'inspirant seulement de celui d'Anselme.

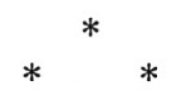

Bien que ces approches modernes aient pu paraître à certains anti-humanistes, en particulier s'agissant de textes spirituels, elles offrent toutefois un point-de-vue très suggestif pour comprendre les processus complexes par lesquels une collection de textes de dévotion entre en relation avec son auditoire au fil des siècles. En acceptant que le $J e$ d'un texte n'appartient pas à l'auteur, mais qu'il est une simple réification de catégories grammaticales, on dégage un outil particulièrement utile pour comprendre par exemple la dynamique textuelle de la prière : le pronom personnel de la première personne, $\mathrm{J} e$, possède une place dans le langage qui peut être occupé par n'importe quel individu. Le $J e$ qui dit les prières et que nous appelons Anselme, peut être Anselme, mais il peut aussi être chaque lecteur pris individuellement qui adopte la place du sujet créé par le langage du texte. Il est certain d'ailleurs, qu'Anselme a eu conscience que son lecteur pouvait s'approprier son langage et convertir ses prières, le Je de l'écrivain devenant celui de son lecteur.

Reste maintenant que les textes que nous lisons sont aussi marqués par le personnage de l'auteur, et que par exemple le langage des Prières ou Méditations dévoilent souvent, derrière l'espérance affichée, des blessures secrètes comme lorsque Anselme, dans la conclusion de la prière à saint Paul, s'adresse au Christ comme à une mère, reflet certain de l'expérience douloureuse d'un enfant privé de toute affection paternelle. On pourrait également s'interroger sur la volonté d'Anselme, conscient de son statut d'auteur et reven-

49 Cf. p. ex. sa préface au Monologion, L'EEuvre de S. Anselme, vol. 1, p. 44-47. 
diquant, contrairement à une longue tradition, la paternité de ces textes de prière, les rassemblant, les publiant et les faisant même enluminer avec beaucoup de soin? Aurions-nous donc parcouru ce long chemin en vain, retrouvant finalement les mêmes analyses et les mêmes réponses ? Je pense plutôt qu'il faut que les spécialistes de l'antiquité et du moyen âge continuent à interroger leurs textes avec les outils mis à leur disposition par la recherche contemporaine : leur lecture s'en trouvera dynamisée et leur compréhension certainement enrichie. 


\section{BIBLIOGRAPHIE}

\section{Sources}

ANSELME DE CANTORBERY, Sancti Anselmi Cantuariensis archiepiscopi opera omnia, éd. dom F. S. SCHMITT, Stuttgart-Bad Canstatt, 1968, 2 t.-6 vol. ; traduction française sous la direction du P. Michel CORBIN, au Cerf, 1986-..., 7 vol. parus. Pour les prières : vol. 5, p. 247431.

AUGUSTIN, Confessions, texte établi et traduit par P. DE LABRIOLLE, Paris, 1925-1937, 2 t. (CUF).

GUIBERT DE NOGENT, Autobiographie, éd. et trad. E.R. LABANDE, Paris, 1981 (CHFMA, 34).

\section{2. Études}

BENTON J.-F., Self and Society in Medieval France. The Memoirs of Abbot Guibert of Nogent (1064-1125), edited with an Introduction and Notes, New YorkEvanston, Harper and Row, 1970.

BENTON J.-F., " Consciousness of Self and Perceptions of Individuality ", in Renaissance and Renewal in the Twelfth Century, R.-L. BENSON-G. CONSTABLE (éd.), Cambridge, Mas., Harvard University Press, 1982, p. 263-95.

BYNUM C.-W., "Did the Twelfth Century discover the Individual ? ", in Journal of Ecclesiastical History, 31, 1980, p. 1-17.

BYNUM C.-W., Jesus as Mother: Studies in the Spirituality of the High Middle Ages, Berkeley, University of California Press, 1982.

COURCELLE P., Connais-toi toi-même : De Socrate à saint Bernard, Paris, Etudes Augustiniennes, 1975, 3 vol. 
CURRIE M., Postmodern Narrative Theory, New-York, St. Martin's Press, 1998 (Transitions).

HANNING R., The Individual in Twelfth-Century Romance, New Haven, Yale University Press, 1977.

JAUSS H.-R., Toward an Aesthetic of Reception, Minneapolis, University of Minnesota Press, 1982.

MORRIS C., The Discovery of the Individual 1050-1200, Londres, SPKC, 1972.

SOUTHERN R.-W., The Making of the Middle Ages, New Haven, Yale University Press, 1953.

SOUTHERN R.-W., Saint Anselm. A portrait in a landscape, Cambridge, 1991 = reprise "revue et augmentée" de Saint Anselm and his biographer. A study of monastic life and thought 1059-c.1130, Cambridge, 1966.

ULMANN W., The Individual and society in the Middle Ages, Baltimore, The Johns Hopkins Press, 1966.

ZINK M., La subjectivité littéraire. Autour du siècle de saint Louis, Paris, PUF, 1985.

ZINK M., Les voix de la conscience. Parole du poète et parole de Dieu dans la littérature médiévale, Caen, Paradigmes, 1992 (Varia, 1).

ZUMTHOR P., Langue, Texte, Enigme, Paris, Seuil, 1975 : ch. 3 «Leje du poète».

ZUMTHOR P., La lettre et la voix. De la «littérature médiévale », Paris, Le Seuil, 1987. 